\title{
NEW ADVANCES ON THE GROTHENDIECK'S INEQUALITY PROBLEM FOR BILINEAR FORMS ON JB*-TRIPLES
}

\author{
AntONio M. PERALTA
}

Abstract. We give a positive answer to the Barton-Friedman's conjecture on "Grothendieck's inequalities" for Cartan factors and $\mathrm{JBW}^{*}$-triples.

Mathematics subject classification (2000): 17C65, 46K70, 46L05, 46L70.

Key words and phrases: Grothendieck's inequalities, JB*-triples.

\section{REFERENCES}

[1] BARTON, T. AND FrIEDMAn, Y., Grothendieck's inequality for JB*-triples and applications, J. London Math. Soc. 362 (1987) 513-523 .

[2] BARTON, T. AND TIMONEY, R. M., Weak*-continuity of Jordan triple products and its applications, Math. Scand. 59, (1986) 177-191.

[3] Chu, C-H., Iochum, B., AND LouPIAS, G., Grothendieck's theorem and factorization of operators in Jordan triples, Math. Ann. 284, (1989) 41-53.

[4] S. DINEEN, The second dual of a JB*-triple system, In: Complex analysis, functional analysis and approximation theory (ed. by J. Múgica), 67-69, (North-Holland Math. Stud. 125), North-Holland, Amsterdam-New York, 1986.

[5] Edwards, C. M., On Jordan $W^{*}$-algebras, Bull. Sci. Math. 104, 2 (1980) 393-403.

[6] FRIEDMAN, Y. AND Russo, B., Structure of the predual of a JBW*-triple, J. Reine u. Angew. Math. 356, (1985) 67-89.

[7] Friedman, Y. And Russo B., The Gelfand-Naimark Theorem for JB * ${ }^{*}$-triples, Duke Math. J. 53, (1986) 139-148.

[8] Grothendieck, A., Résumé de la théorie métrique des produits tensoriels topologiques, Bol. Soc. Mat. Sao Paolo 8, (1956) 1-79.

[9] HaAgerup, U., Solution of the similarity problem for cyclic representations of C*-algebras, Ann. of Math. 118, (1983) 215-240.

[10] HaAgerup, U., The Grothendieck inequality for bilinear forms on C*-algebras, Avd. Math. 56, (1985) 93-116.

[11] Hanche-Olsen, H. And StøRmer, E., Jordan operator algebras, Monographs and Studies in Mathematics 21, Pitman, London-Boston-Melbourne 1984.

[12] Ho, T., Martínez-Moreno J., Peralta, A. M. And Russo, B., Derivations on real and complex $J B^{*}$-triples, J. London Math. Soc. 65 2, (2002) 85-102.

[13] KAUP, W., Über die Klassifikation der symmetrischen hermiteschen Mannigfaltigkeiten unendlicher Dimension. I, Math. Ann. 257, 4 (1981), 463-486.

[14] KAUP, W., A Riemann mapping theorem for bounded symmetric domains in complex Banach spaces, Math. Z. 183, (1983) 503-529.

[15] Peralta, A. M., Little Grothendieck's theorem for real JB*-triples, Math. Z., 237, 3 (2001) 531-545.

[16] Peralta, A. M. AND RodrígueZ Palacios, A., Grothendieck's inequalities for real and complex JBW $^{*}$-triples, Proc. London Math. Soc. (3) 833 (2001), 605-625.

[17] Peralta, A. M. And Rodríguez Palacios, A., Grothendieck's inequalities revisited, Recent progress in functional analysis (Valencia, 2000), 409-423, North-Holland Math. Stud., 189, North-Holland, Amsterdam, 2001. 
[18] PISIER, G., Grothendieck's theorem for non commutative $C^{*}$-algebras with an appendix on Grothendieck's constant, J. Funct. Anal. 29, (1978), 397-415.

[19] RodRíGUEZ A., On the strong* topology of a JBW*-triple, Quart. J. Math. Oxford 422 (1989), 99-103.

[20] SAKAI, S.: $C^{*}$-algebras and $W^{*}$-algebras, Springer-Verlag, Berlin 1971.

[21] Wright, J. D. M., Jordan C*-algebras, Michigan Math. J. 24, (1977) 291-302. 